\title{
Mamogram Image Classification Using Extreme Learning Machine
}

\author{
S. Dhivya, A. Nithya and T. Abirami \\ Department of Electronics and Communication Engineering, M. Kumarasamy College of Engineering, Karur - \\ 639113, Tamil Nadu, India; dhivyas.ece@mkce.ac.in, nithyaa.ece@mkce.ac.in , abiramit.ece@mkce.ac.in
}

\begin{abstract}
Objective: To make early diagnosis of breast cancer from mammogram image. Methods/Statistical analysis: To avoid the misdiagnosis, we proposed a system to sort the suspicious masses from the mammogram image by using Extreme learning machine algorithm. The ELM based classifier is used to classify the input data as malignant and benign classes with the abnormal class. The effectiveness of the ELM algorithm is superior to the other existing algorithms for mammogram classification problems with its reduced training time and classification accuracy. Findings: We provide an optimistic method for binary class classification of mammograms using extreme learning machine algorithm. Mammography is a technique which is preferred for early diagnosis of breast cancer. On the other hand, in most cases, it is not easy to differentiate benign and malignant tumor without biopsy, hence misdiagnosis is always possible. The machine learning algorithm provides high accuracy than other techniques and also the execution time is very low when compared to normal diagnosis. The existing methods are very slow compared to this proposed technique. The input images are the mammogram image and the segmentation, preprocessing is performed to remove the noises present. Application/Improvements: The main application of the system is the early diagnosis of cancerous cell present and also classifies the normal and abnormal images.
\end{abstract}

Keywords: Breast Cancer, Extreme Learning Machine, Mammography

\section{Introduction}

There are many screening methods for breast cancer diagnosis viz., MRI, Mammogram and Ultrasound etc. Among them, Mammogram may be the superior means for detecting breast cancer. But it does not diagnosis the cancer at early stage; so the physical examinations still prevails. In modern existence, the occurrence rate of breast cancer has significantly augmented $\stackrel{1}{-}$. But, biopsy is the only way to identify whether detected tumor is benign or malignant, which is an invasive procedure that eliminates the tumor cells or tissue from a patient. A noninvasive method of detecting abnormalities in mammograms can diminish the need for needless biopsies, which reduces the patients of trouble and reduction of medical expenses.

All together, breast cancer endurance rate is enhanced over the past few years with the advancement of more efficient investigative methods and growth in healing methodologies. The American Cancer Society had anticipate that about 230480 new cases of persistent breast cancer and over 57,650 new cases of noninvasive breast cancer has been analyzed in the United States in 2011 and nearly 39,520 women would expire due to breast cancer ${ }^{2}$. The highly accepted analytical method known as mammogram uses $\mathrm{X}$-rays of low dose, high contrast and high-resolution detectors. An X-ray system designed exclusively to image the breasts is the mammography method. Mammogram is used for screening and analysis of breast cancer. Screen Film Mammography (SFM) and Full-Field Digital Mammography (FFDM) are the two types of mammography screening system used for diagnosis. SFM uses a film screen as a final recording device and FFDM, uses digital detectors as the recording media. The digital images obtained by FFDM have many advantages than SFM which produce a processed and enhanced image $\frac{3-6}{6}$. 


\section{Proposed Methodology}

In the proposed system the input image is gathered from the mammography with a dataset of mammograms given by the Singapore Anti-Tuberculosis Association CommHealth, Singapore. Test input from mammograms are taken from normal, benign, and cancerous breasts. The entire test images are taken from victims of age group 45 to 70 years. Test inputs are collected and they are processed to obtain a resolution of $1024 \times 1680$. Every sample is processed in two views namely the cranio-caudal view and the mediolateral-oblique view. From the proposed system, the images which are number is larger when compared with the abnormal images with a ratio of 223:90 in which the abnormal image consist of both benign and malignant which provides an high accurate result based on ELM classification.

Before classifying the image preprocessing, segmentation and feature extraction are done and that image is then send for classification to get high accuracy and less error rate.

Figure 1 describes about the steps involved in the process.

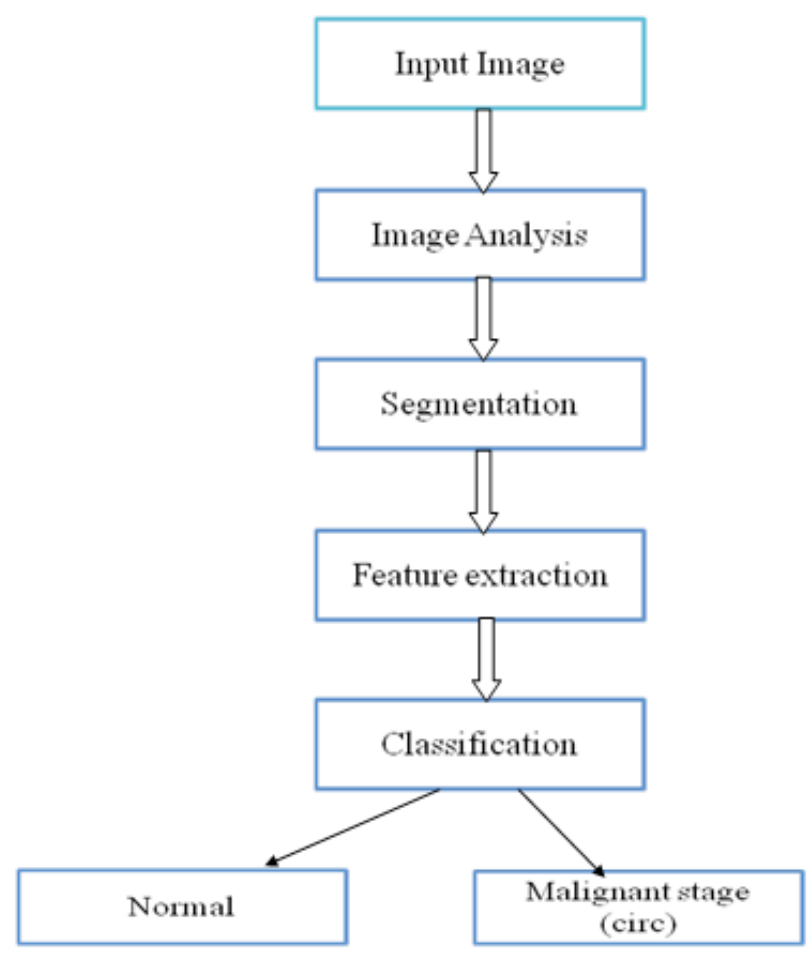

Figure 1. The flow chart of the proposed method.

\subsection{Input Image}

Before gathering the info information, arrangements of prepared pictures are gathered and are stacked in the classifier. At that point the information pictures are gathered from the dataset for additionally preparing. The gathered pictures are won't be in same pixel measure so it is hard to process the picture. For that the gathered pictures are changed over into standard pixel esteem either as $512 \times$ 512 or $640 \times 480$ for simple handling of information.

\subsection{Preprocessing}

Images are effectively get influenced by the clamor introduce in the framework or because of the outside environment. The different kinds of clamors introduce in restorative pictures are Gaussian commotion, salt and pepper clamor, Poisson clamor and so forth. These commotions will lessen the execution. So legitimate denoising must be performed. Commotions can be expelled either by sifting or by thresholding. In this paper middle channel is utilized. It is a sort of non straight channel which is utilized to decrease the power variety in the pictures. In middle separating the pixel estimation of the picture is supplanted by the area middle esteem.

\subsection{Segmentation}

Segmentation is the technique for isolating the picture into required parts. It is performed to find the required limit of a picture which is utilized for grouping. The pixels which are of same power, shading or surface are divided into a gathering. Diagram cut strategy is utilized for portioning the picture. The chart cut calculation basically in view of max stream/min-cut enhancement. Diagram cut calculation will suits best for Binary arrangement. Minimization is performed utilizing min-cut calculation. For max stream Boykov-Kolmogorov is the proficient calculation. It is an iterative procedure and the procedure is rehashed until the point when the merging is accomplished. In iterative diagram the improvement is done in light of hues by utilizing $\mathrm{k}$ implies group.

\subsection{Feature Extraction}

The component extraction is utilized to separate or select the base measure of pixel esteem from the vast contribution by disposing of the excess esteem. The required highlights are removed by utilizing this procedure. The element extraction strategy incorporates nearby par- 
allel examples, Haar wavelets, Histogram of Oriented Gradients (HOG), Speeded Up Robust Features (SURF), shading histogram. The method utilized as a part of this paper is neighborhood twofold examples. Nearby paired examples (trim) are for the most part utilized for surface arrangement of pictures. The picture to be inspected is isolated into number of cells with pixel esteem. At that point the every pixel in the cells is: contrasted and the area pixel, histogram is acquired, and the gotten histogram is then standardized which gives an element vector.

\subsection{Classification}

The last procedure is the characterization of the extricated pictures to distinguish the sick picture. Outrageous Learning Machine classifier is utilized to group the extricated picture. Extraordinary Learning Machine classifier is a regulated machine learning calculation. The arrangement of the picture is made with the prepared information which is as of now exhibit in the informational collection. Extraordinary Learning Machine (ELM) is a Solitary concealed Layer nourishes Forward Neural Network (SLFNN) system which at irregular chooses input weights and shrouded neuron predispositions without preparing. The yields weights are systematically computed utilizing the standard minimum square arrangement and MoorePenrose reverse of a general straight framework, which permits a noteworthy lessening in preparing time. The initiation work like sine, Gaussian, sigmoidal and so forth, can be decided for concealed neuron layer and straight enactment capacities for the yield neurons ${ }^{7}$. The SLFNN assessed here utilizations added substance neuron outline rather than part based, henceforth irregular parameter determination. SLFNs are considered as a direct framework. The one of a kind least standard minimum square (LS) arrangement is demonstrated as

$$
\hat{\beta}=\mathrm{H}^{+} \mathrm{T}
$$

\section{Results}

The input image has been processed and classified result obtained by using ELM classification algorithm in our proposed system is shown in Figure 2. The test image is compared with the trained image and the result produced is either normal or abnormal image which represents the benign or malignant cancer or not.

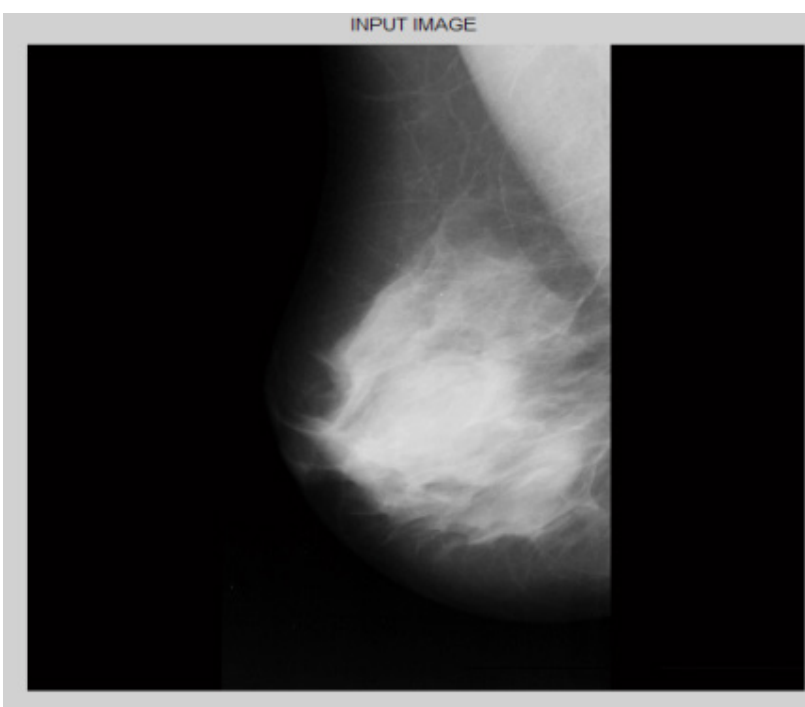

(a)

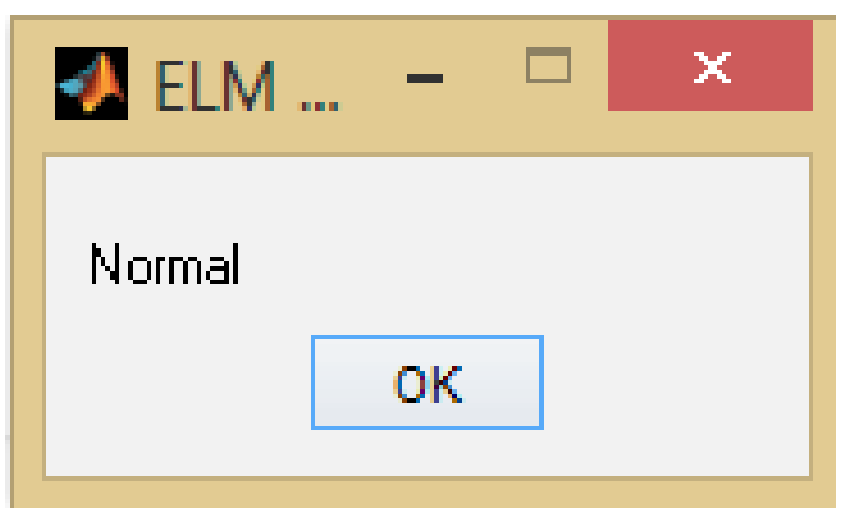

(b)

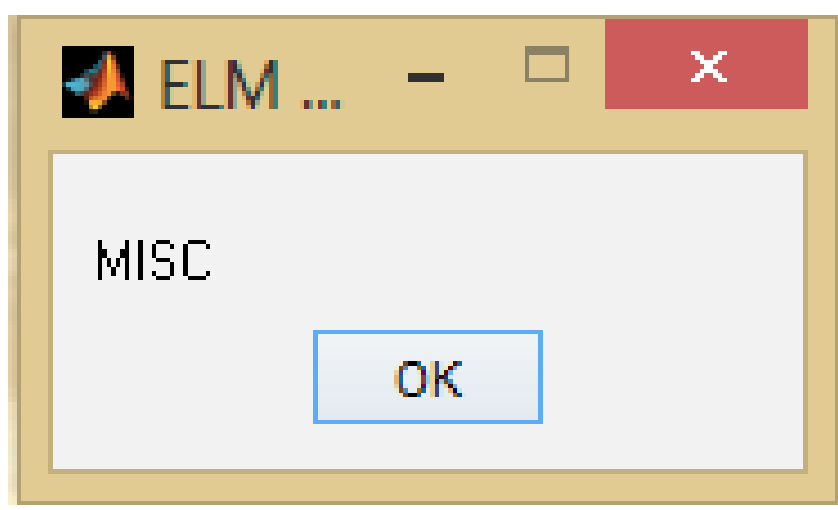

(c)

Figure 2. (a) Input image, (b) Normal output image after classification, and (c) Abnormal output image after classification. 


\section{Conclusion}

The main motive of the proposed system is to classify the cancer cell from the mammogram image. The images are classified using extreme learning machine based classifier as malignant and benign classes with the abnormal class. The overall system accuracy achieved is around $92 \%$ and the elapse time of the algorithm ranges between 0.0020.019 seconds. The efficiency of the ELM classifier is more efficient when compare with other existing classifier algorithms for mammography image classification problems with its reduced training time and classification accuracy to classify the image according to its features and to group them in the malignant, benign and normal groups. The future work is to implement the process with the new developing E2LM algorithm which is much superior algorithm to every other algorithm available in artificial intelligence algorithms.

\section{References}

1. Polat K, Genes S. Breast cancer diagnosis using least square support vector machine, Digital Signal Processing. 2007; 17(4):694-701. Crossref.
2. Tax D, Duin R. Uniform object generation for optimizing one-class Classifiers, Journal of Machine Learning Research. 2001; 2:155-73.

3. Pisano ED, Hendrick RE, Yaffe MJ, Baum JK, Acharyya S, Cormack JB. Diagnostic accuracy of digital versus film mammography: Exploratory analysis of selected population subgroups in DMIST, Radiology. 2008; 246(2):376-83. Crossref. PMid: 18227537, PMCid: PMC2659550.

4. Mencattini A, Salmeri M, Lojacono R, Frigerio M, Caselli F. Mammographic images enhancement and denoising for breast cancer detection using dyadic wavelet processing, IEEE Transactions on Instrumentation and Measurement. 2008; 57(7):1422-30. Crossref.

5. Schulz-Wendtland R, Fuchsjigerb $M$, Wackerc $T$, Hermannd KP. Digital mammography: An update, European Journal of Radiology. 2009; 72(2):258-65. Crossref. PMid: 19592186.

6. Pisano ED, Gatsonis C, Hendrick RE, Yaffe MJ, Baum JK, Acharyya S, Cormack JB. Diagnostic performance of digital versus film mammography for breast-cancer screening, The New England Journal of Medicine. 2005; 353(17):1773-83. Crossref. PMid: 16169887.

7. Deepa SN, Arunadevi B. Extreme learning machine for classification of brain tumor in 3DMR images, Informatol. 2013; 46(2):111-21. 